\title{
Bioengineering Methods in MicroRNA-Mediated Direct Reprogramming of Fibroblasts Into Cardiomyocytes
}

\author{
Camilla Paoletti* and Valeria Chiono \\ Department of Mechanical and Aerospace Engineering, Politecnico di Torino, Turin, Italy
}

Ischemic heart disease is the major cause of mortality worldwide. Despite the most recent pharmacological progresses, cardiac regeneration is yet not possible, and heart transplantation is the only therapeutic option for end-stage heart failure. Traditional cardiac regenerative medicine approaches, such as cell therapies and tissue engineering, have failed in the obtainment of human functional cardiac tissue, mainly due to unavailability of high quantities of autologous functional cardiomyocytes (CMs), low grafting efficiency, and/or arrhythmic events. Direct reprogramming (DR) of fibroblasts

OPEN ACCESS

Edited by:

Sveva Bollini,

University of Genoa, Italy

Reviewed by:

Francesca Bortolotti, International Centre for Genetic Engineering and Biotechnology, Italy Anna Marsano,

University of Basel, Switzerland

*Correspondence:

Camilla Paoletti camilla.paoletti@polito.it

Specialty section: This article was submitted to Cardiovascular Biologics and

Regenerative Medicine,

a section of the journal

Frontiers in Cardiovascular Medicine

Received: 30 July 2021 Accepted: 13 September 2021

Published: 25 October 2021

Citation:

Paoletti C and Chiono V (2021) Bioengineering Methods in MicroRNA-Mediated Direct Reprogramming of Fibroblasts Into Cardiomyocytes.

Front. Cardiovasc. Med. 8:750438.

doi: 10.3389/fcvm.2021.750438 into induced CMs (iCMs) has emerged as a new promising approach for myocardial regeneration by in situ transdifferentiation or providing additional CM source for cell therapy. Among available DR methods, non-viral transfection with microRNAs (miRcombo: miR-1, miR-133, miR-208, and miR-499) appears promising for future clinical translation. MiRcombo transfection of fibroblasts could be significantly improved by the development of safe nanocarriers, efficiently delivering their cargo to target cells at the required stoichiometric ratio and overall dose in due times. Newly designed in vitro 3D culture microenvironments, providing biomimetic biophysical and biochemical stimuli to miRcombo-transfected cells, significantly increase the yield of fibroblast transdifferentiation into iCMs, enhancing CM gene expression. Epigenetic regulation of gene expression programs, critical to cell lineage commitment, can also be promoted by the administration of specific anti-inflammatory and anti-fibrotic soluble factors, helping in suppressing fibroblast signature. The aim of this mini-review is to introduce the readers to a relatively unknown field of cardiac research integrating bioengineering tools as relevant for the progress of miRNA-mediated cardiac DR.

Keywords: myocardial infarction, microRNAs, fibroblasts, cardiomyocytes, cell reprogramming, tissue engineering, nanoparticles

\section{INTRODUCTION}

Ischemic heart disease is a major cause of mortality with more than 23 million cases worldwide $(1,2)$. During myocardial infarction (MI), billions of cardiomyocytes (CMs) are irreversibly lost and replaced by cardiac fibroblasts (CFs) forming a non-contractile scar tissue, which undergoes continuous remodeling, causing left ventricle dilation and progressive heart failure $(3,4)$. Given the poor endogenous regenerative potential of the adult heart, recovery of cardiac functionality could be accomplished by the replenishment of lost CMs. However, cell therapies and cardiac 
tissue engineering strategies have achieved limited success due to poor engrafting, survival, and integration of implanted cells, alone or in combination with biomaterials, into the host tissue and the unmet need for a source of mature and functional CMs (1-4).

Direct reprogramming (DR) of fibroblasts into induced CMs (iCMs) has emerged as a new source for CMs (5-7). Previous literature has reported several different cardiac DR strategies, including the upregulation of cardiac transcription factors (TFs) (8), the administration of complex combinations of small molecules (9), and the modulation of microRNAs (miRNAs) (6). MiRNAs are short non-coding RNAs (of $\sim 21$ nucleotides) that regulate gene expression post-transcriptionally (10).

Despite excitement on DR potentialities for cardiac regeneration, the approach is in need of optimization. Main limitations include the low yield of fibroblast DR into iCMs, the wide use of unsafe viral vectors, and the generation of predominantly immature, partially reprogrammed iCMs $(11,12)$.

The present mini-review focuses on miRNA-mediated DR of fibroblasts into iCMs as a promising approach for future translation of cardiac DR into clinical settings. Herein, we discuss the key role of bioengineering research in improving cardiac DR efficiency and $\mathrm{iCM}$ maturation, through the design of efficient and safe miRNA-releasing nanocarriers and biomimetic in vitro culture microenvironments (Figure 1).

\section{NON-VIRAL MicroRNA DELIVERY SYSTEMS FOR DIRECT REPROGRAMMING}

\section{MicroRNA-Mediated Reprogramming of Fibroblasts Into Cardiomyocytes}

The use of miRNAs for cardiac DR was first studied in 2012 by Jayawardena et al. (6). An accurate screening of CM-specific miRNAs allowed the selection of six miRNA candidates involved in CM differentiation and development. A minimal combination of four miRNAs called miRcombo (miR-1, miR-133, miR-208, and miR-499) was then identified as able to promote DR of mouse fibroblasts into iCMs $(6,7)$. The role of such miRNAs in cardiac development was subsequently reported by several studies (13). MiR-1 and miR-133 are co-transcribed in the cardiac and skeletal muscle tissues during embryonic development, and their expression increases until adulthood (13). MiR-1 is involved in regulating CM proliferation and ventricular organization. MiR133 shares common functions with miR-1. Conversely, miR-208 and miR-499 mostly regulate the expression of $\alpha$ and $\beta$ isoforms of myosin heavy chain (MHC), which are involved in CM contraction (13). Transient transfection with miRcombo, using a commercial transfection agent (DharmaFECT ${ }^{\mathrm{TM}}$ ), was sufficient to induce in vitro $\mathrm{DR}$ of mouse neonatal and adult fibroblasts into iCMs $(6,14)$. Transfected cells expressed CM genes and proteins, showing sarcomeric organization and spontaneous calcium oscillations. Moreover, miRcombo delivery using a lentivirus induced in vivo DR of fibroblasts into iCMs (14).
Initially, microRNA delivery and upregulation of cardiac TFs were combined to induce DR of human fibroblasts into iCMs in mixed viral/non-viral approaches $(15,16)$. A reprogramming cocktail consisting of four TFs (Gata4, Tbx5, Hand2, and Myocardin) and two miRNAs (miR-1 and miR-133) was the most efficient in inducing DR of human foreskin fibroblasts (HFFs), adult human dermal fibroblasts (AHDFs), and adult human CFs (AHCFs) into iCMs (15).

However, in 2020, Paoletti et al. demonstrated that non-viral transient transfection with miRcombo (using DharmaFECT) is enough to trigger the in vitro transdifferentiation of AHCFs into iCMs (7). MiRcombo-transfected AHCFs showed enhanced expression of cardiac TFs (Gata4, Mef2c, Tbx5, and Hand2) at 7 days post-transfection, while at 15 days, $11 \%$ of cells expressed cardiac troponin $\mathrm{T}$ (cTnT), and at 30 days, $38 \%$ of cells showed spontaneous calcium oscillations (7).

Recently, miRcombo-mediated cardiac DR efficiency was improved by transfecting mouse fibroblasts with a polycistronic vector, inducing equivalent expression levels of the four microRNAs of miRcombo (17).

Recently, the administration of a polycistronic vector inducing equivalent expression levels of miRcombo was found to improve efficiency of mouse fibroblasts DR into iCMs (17), suggesting the need for delivery vectors, ensuring miRcombo delivery at a stoichiometric ratio.

\section{Alternative Strategies for MicroRNA Delivery in Direct Reprogramming}

In the last years, alternative non-viral strategies to lipid nano-formulations have been proposed for miRNA delivery. Biomaterial-based nanoparticles (NPs) should efficiently encapsulate miRNAs, protecting them from rapid degradation and ensuring their efficient release to target cells, through specific ligands on NP surface for receptor-mediated endocytosis (18). Synthetic polymers [e.g., poly(lactic-co-glycolic acid) (PLGA); poly(ethylene glycol) (PEG); and poly(ethylenimine) (PEI)], natural polymers (e.g., chitosan), and inorganic materials (e.g., gold and calcium phosphate) have been investigated as biomaterials for miRNA-loaded nanocarriers (18). Muniyandi et al. have proposed PLGA/PEI NPs encapsulating miR-1 and miR-133 for DR of AHCFs into iCMs (19). PLGA/PEI NPs showed cytocompatibility and $\mathrm{pH}$-dependent payload release and induced the expression of structural ( $\alpha$-sarcomeric actinin) and functional (cTnT) CM proteins at 7 days posttransfection (19). Recently, Yang et al. have reported that branched-PEI coated nitrogen-enriched carbon dots (BP-NCDs) can encapsulate miRcombo for DR of neonatal mouse CFs into iCMs in vitro and in vivo (20). BP-NCDs efficiently delivered miRNAs to target cells, inducing the expression of specific CM genes (Nppa, Nkx2.5, and Myh7) and proteins (Gata4, Mef2c, Hand2, and Tbx5) in vitro. Furthermore, in situ delivery of miRcombo by BP-NCDs in an MI mouse model significantly reduced the infarcted area after 4 weeks compared with control groups. On the other hand, limitations of this study derive from the testing of neonatal mouse CFs rather than adult human 


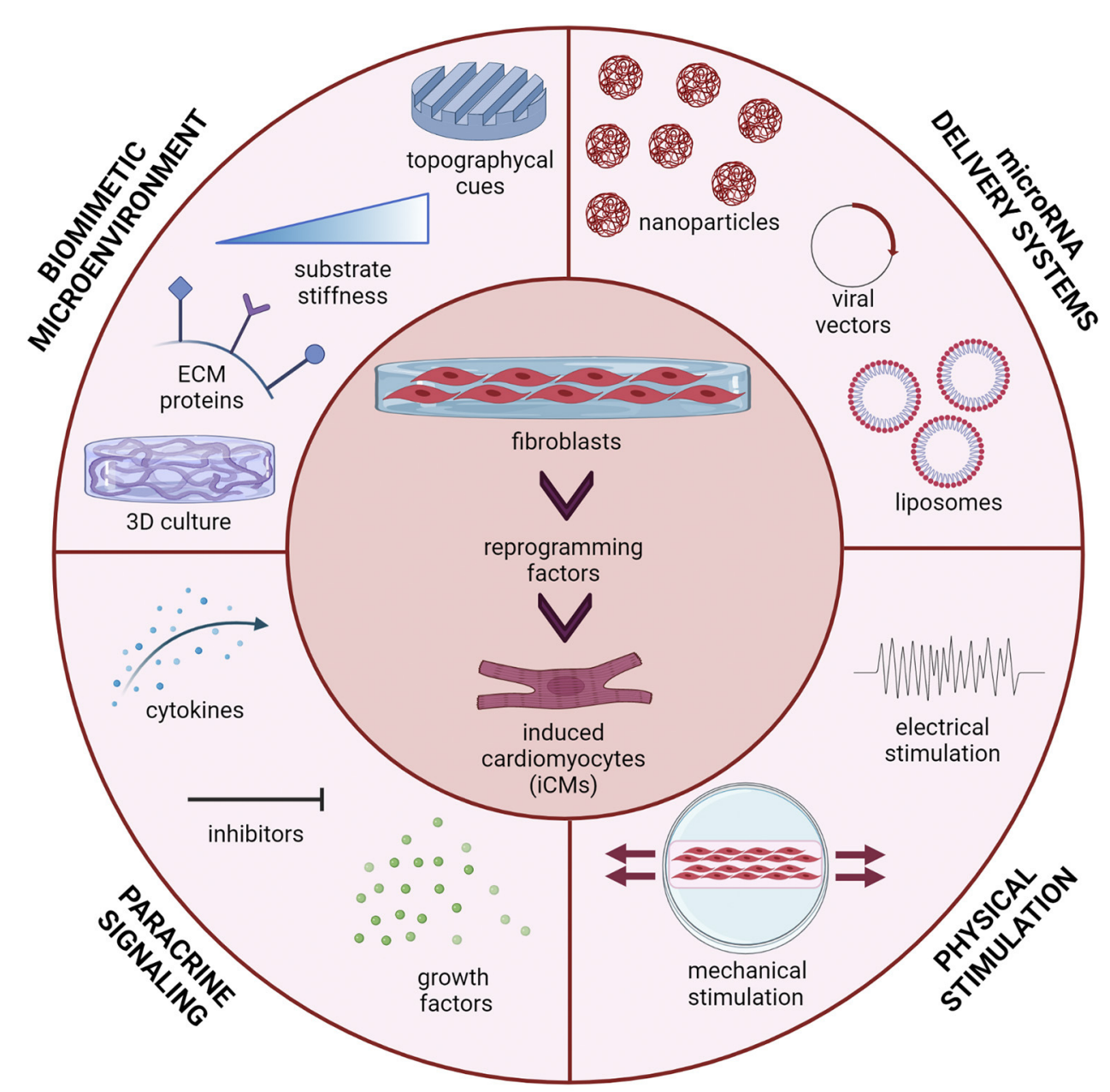

FIGURE 1 | Multiple stimuli can affect direct reprogramming (DR) of fibroblasts into induced cardiomyocytes (iCMs): microRNA delivery strategy (viral vectors, liposomes, polymeric nanoparticles); the microenvironment in which cells are cultured (three-dimensional culture, topographical cues, substrate stiffness and extracellular matrix proteins); paracrine signals (cytokines, inhibitors and growth factors) and physical stimuli (mechanical stretching, electrical stimulation). Figure was created using Biorender.com.

fibroblasts, the lack of extensive characterization of BP-NCDs in terms of encapsulation ability and release kinetics, and the non-degradability of the nanocarriers, which is associated with long-term safety concerns.

Hence, optimal nanocarriers for miRcombo delivery to human adult fibroblasts are currently missing. Furthermore, fibroblast specific targeting with functionalized NPs (e.g., using peptides, antibodies, or aptamers) could increase NP specificity of cargo release, thus maximizing DR effects and reducing offtarget effects (21). However, specific ligands that recognize only fibroblasts populating the fibrotic scar are still under study.

\section{BIOMIMETIC CULTURE MICROENVIRONMENT}

Previous literature has reported higher DR yield achieved during in vivo experiments in mouse model compared with in vitro 2D cultures. This finding suggests that a three-dimensional
(3D) culture microenvironment mimicking the biophysical and biochemical properties of cardiac tissue (Table 1) has the potential to significantly improve cardiac DR outcomes (13). In vitro culture of miRNA-transfected fibroblasts in cardiac tissue mimetic microenvironments may enhance DR efficiency and iCM maturation, as discussed in the next paragraphs (Table 2).

\section{Cell-Substrate Interactions: Biochemical and Biophysical Properties of the Culture Substrate}

In 2016, Sia et al. studied DR of neonatal tail tip fibroblasts (TTFs) transfected with retroviruses expressing Gata4, Mef2c, and Tbx5 (GMT). After transfection, the cells were seeded on Matrigelcoated polyacrylamide hydrogels with different stiffness (from 1 to $62 \mathrm{kPa})$. Reprogramming yield after 10 days of culture $(\sim 17 \%)$ did not vary with substrate stiffness (27). On the contrary, microgroove culture substrates increased the yield $(\sim 30 \%)$ of fibroblasts DR into iCMs. Cells showed sarcomere structures and 
TABLE 1 | Reference cardiac tissue-like properties for biomimetic culture microenvironment.

\begin{tabular}{|c|c|c|}
\hline Properties & Reference values in cardiac tissue & References \\
\hline $\begin{array}{l}\text { Human cardiac } \\
\text { ECM composition }\end{array}$ & $\begin{array}{l}70 \% \text { fibrillar collagen (collagen I and V), } \\
20 \% \text { basement membrane (collagen IV, } \\
\text { laminin, agrin, perlecan, nidogen), } 4 \% \\
\text { structural ECM (proteoglycans and fibrous } \\
\text { glycoproteins), 3\% matricellular } \\
\text { components (collagen VI, fibronectin) }\end{array}$ & $(22)$ \\
\hline Stiffness & $\begin{array}{l}1-6 \mathrm{kPa} \text { (fetal); } 10-15 \mathrm{kPa} \text { (adult); > } 50 \\
\mathrm{kPa} \text { (fibrotic) }\end{array}$ & (23) \\
\hline $\begin{array}{l}\text { Anisotropic ratio of } \\
\text { stiffness }\end{array}$ & $1.9-3.9$ & $(24)$ \\
\hline $\begin{array}{l}\text { Cyclic mechanical } \\
\text { deformation }\end{array}$ & $\begin{array}{l}1 \mathrm{~Hz} \text { (in humans), } 10 \% \text { at early stage of } \\
\text { diastole (stiffness: } 10-20 \mathrm{kPa} \text { ) up to } 15-22 \\
\% \text { at the end of diastole (stiffness: } 50 \mathrm{kPa} \text { ) }\end{array}$ & $(24)$ \\
\hline $\begin{array}{l}\text { Electrical } \\
\text { conductivity }\end{array}$ & $0.57 \mathrm{~S} / \mathrm{m}$ & $(24)$ \\
\hline $\begin{array}{l}\text { Topographical } \\
\text { cues }\end{array}$ & $\begin{array}{l}\text { In native heart, myocardial fibers are } \\
\text { arranged into distinct laminae (4-6 } \\
\text { myocytes thick) separated by } \\
\text { collagen-based ECM: helical-laminar } \\
\text { assembly of hierarchically organized } \\
\text { fibrillar structures. }\end{array}$ & $(25)$ \\
\hline
\end{tabular}

spontaneous contractile activity, attributed to higher expression of Mkl1, a mechanosensitive TFs, and histone $\mathrm{H} 3$ acetylation for chromatin remodeling (27).

More recently, embryonic mouse fibroblasts, transfected with Gata4, Mef2c, Tbx5, and Hand2 (GMTH), were cultured on Matrigel-conjugated polyacrylamide hydrogels with different stiffnesses (1-126 kPa). Higher DR efficiency was obtained on substrates with similar stiffness $(8 \mathrm{kPa})$ to healthy myocardium, compared with rigid polystyrene dishes $(\sim \mathrm{GPa})$. This result was attributed to the suppression of YAP/TAZ (Yes-associated protein/transcriptional coactivator with PDZ-binding domain) signaling and silencing of fibroblast gene programs, induced by a culture microenvironment with biomimetic stiffness (28). With respect the previous work by Sia et al. (27), the more efficient protocol for cardiac DR by GMTH transfection and the use of embryonic fibroblasts could account for the superior DR efficiency, despite the use of similar culture substrates. Although this result suggests variability of DR outcomes depending on fibroblasts types and reprogramming protocol, the role of mechanosensing was outlined. However, both studies were limited by the investigation of $2 \mathrm{D}$ cell cultures on the surface of hydrogels or microgroove substrates.

DR of fibroblasts embedded in 3D biomimetic matrices was only studied by Li et al. (26). In their work, miRcombotransfected mouse fibroblasts cultured into 3D fibrin/Matrigel hydrogels showed higher DR efficiency compared with 2D cultures, as suggested by the higher expression of CM genes ( $\alpha$-MHC, cardiac troponin I, $\alpha$-sarcomeric actinin, and Kcnj2) and proteins (cardiac troponin I and $\alpha$-sarcomeric actinin). Such result was attributed to the upregulation of specific matrix metalloproteinases when cells were embedded in 3D hydrogels (26). Notably, 3D cell culture alone was sufficient to enhance the expression of CM TFs in non-transfected mouse fibroblasts compared with $2 \mathrm{D}$ cell cultures, suggesting that $3 \mathrm{D}$ culture microenvironment itself can promote the expression of $\mathrm{CM}$ genes (26).

Beyond biophysical characteristics of culture substrates, biochemical cues, such as proteins of the cardiac extracellular matrix (cECM), can help in recreating similar in vitro culture conditions to in vivo microenvironment (36). Indeed, in a different application, a 3D microenvironment containing brain ECM (bECM) was found to boost fibroblast DR into induced neuronal cells (iNs) (37). In this regard, gene set enrichment analysis (GSEA) of mouse embryonic fibroblasts (MEFs) transduced with MGT (Mef2c-Gata4-Tbx5) plasmids have shown that CECM proteins, such as collagens and laminins, are already expressed after 48 and $72 \mathrm{~h}$ posttransduction (38). Such findings suggest that in the early stages of fibroblast reprogramming, cells naturally create a suitable microenvironment, which enhances transdifferentiation. Indeed, Smith et al. have designed culture substrates based on PEG hydrogels functionalized with a high concentration of laminin and RGD peptide, achieving more efficient DR of mouse fibroblasts into iCMs, compared with hydrogels with low concentrations of RGD adhesion motifs or tissue culture polystyrene surfaces (30).

\section{Paracrine Signaling and Small Molecules}

Fibroblast DR in vivo is influenced by innumerable extrinsic factors of the cardiac microenvironment, encompassing not only mechanical forces or topographical cues but also the presence of cytokines, growth factors, and paracrine signals in the heart. After MI, pro-inflammatory cytokines are released in the wounded area, supporting cardiac remodeling through immune cells and fibroblast recruitment, inducing the deposition of stiffer ECM (39). Given the key roles that cytokines play during MI, it is worth studying how cytokines may influence cell reprogramming. Enrichment analysis of pathways that regulate cardiac reprogramming showed that anti-inflammatory cytokines (IFNA2, IFNA16, and IL10) are positively associated with DR and are called "activators," while pro-inflammatory molecules (IL1A, IL2, and IL26 cytokines and TF CEBP $\beta$ ) were mostly identified as "inhibitors" (31). Indeed, TF ZNF281 was found to enhance cardiac DR via downregulation of genes involved in inflammatory response. Similarly, Testa et al. showed that treatment of mouse CFs with PTC-209, a Bmil inhibitor, before DR, negatively affected STAT3 and ERK1/2 phosphorylation, improving DR of fibroblasts into iCMs via inhibition of inflammatory pathways (32). Additionally, Jayawardena et al. found that JAK/STAT pathway suppression using Jak Inhibitor I, combined with miRcombo, enhanced DR of mouse fibroblasts into iCMs in vitro (6). Small molecule diclofenac, an inhibitor of cyclooxygenase-2 (COX-2) signaling, was also found to significantly enhance DR via PGE2/EP4 suppression, inducing sarcomere organization and increased number of beating cells as compared with GHMT alone in TTFs (33).

Moreover, a potential approach for improving DR relies also on inhibiting fibroblast endogenous signaling pathways 
TABLE 2 | Selected bioengineering studies on DR of fibroblasts into iCMs.

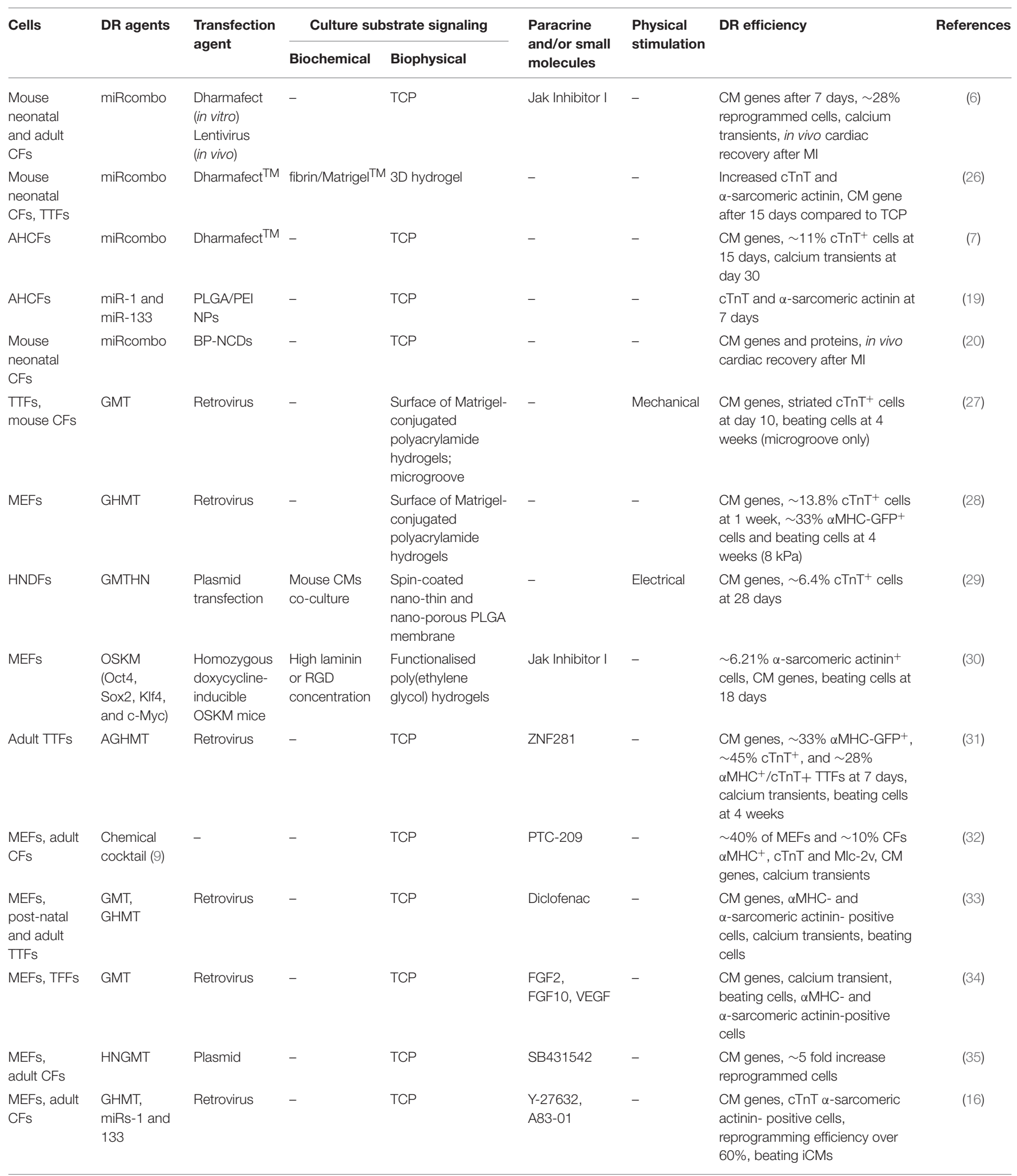

This table shows differential DR strategies for iCM generation combining cell source, reprogramming factors and delivery methods, biomimetic microenvironment, paracrine signaling and/or inhibitors and physical stimuli. DR efficiency reports the expression of cardiomyocyte genes and protein, electrophysiological characters and beating property. CFs cardiac fibroblasts, MEFs mouse embryonic fibroblasts, TTFs tail-tip fibroblasts, AHCFs adult human cardiac fibroblasts, HNDFs human neonatal dermal fibroblasts, $\alpha$-MHC $\alpha$-myosin heavy chain, CTnT cardiac troponin T, CM cardiomyocyte, TCP tissue culture polystyrene. 
that maintain fibroblast identity. Silencing of transforming growth factor-beta (TGF- $\beta$ ) and rho-associated kinase (ROCK) signaling combined with different reprogramming cocktails was reported to improve DR. The use of TGF- $\beta$ inhibitor SB431542 in combination with GHMT in mouse embryonic and adult fibroblasts was reported to induce $\sim 5$-fold increase in cell reprogramming after 10 days of culture (35). Moreover, Zhao et al. have reported that DR is enhanced in GHMTtransfected mouse fibroblasts by overexpressing miR-1 and miR133 combined with ROCK or TGF- $\beta$ inhibitors, suggesting a synergistic effect in overcoming reprogramming barriers (16).

Yamakawa et al. have studied MEF reprogramming into iCMs in defined serum-free medium containing fibroblast growth factor (FGF) 2, FGF10, and vascular endothelial growth factor (VEGF) (34). The addition of these growth factors after cell transduction with Mef2c and Tbx5 successfully generated iCMs, exhibiting calcium oscillation and spontaneous contraction, by activating cardiac transcriptional regulators, including Gata4. Defined culture conditions influenced cardiac DR only in the later stage of transdifferentiation (34).

\section{Further Physical Stimulations: Cyclic Mechanical Stretching and Electrical Stimulations}

Mechanical stimulation of cultured induced pluripotent stem cell (iPSC)-derived CMs was found to enhance cellular alignment and sarcomere organization, calcium handling, and contractile properties, causing alterations in gene and protein expression toward a mature phenotype (40). As described above, Sia et al. have investigated different biophysical stimuli to induce fibroblast DR. Mechanical cyclic stimulation (10\% strain, 1$\mathrm{Hz}$ frequency) applied for 10 days reduced the percentage of reprogrammed cells compared with static cultures (27). Under mechanical stimulation, the expression of hallmarks of fibrotic scar tissue (collagen I and fibronectin) and reinforcement of fibroblast signature could account for the detected decrease in DR yield (41). On the other hand, mechanical cyclic stretching applied at a later stage of cardiac DR could potentially improve iCM maturation, as suggested by the wide literature on iPSC differentiation into CMs (42).

Electrical stimulation was also tested in cardiac DR, considering its beneficial effect on maturation of stem cell-derived CMs (43). Heart-like electric stimulation (1 $\mathrm{V} / \mathrm{cm}$, biphasic square pulse for $5 \mathrm{~ms}$ at $5 \mathrm{~Hz}$ ) of GMTHN (Gata4, Mef2c, Tbx5, Hand2, and Nkx2.5) transfected human neonatal dermal fibroblasts (HNDFs), cocultured with murine CMs on nano-thin and nano-porous PLGA membranes, significantly increased DR yield, inducing the expression of CM genes and increasing the percentage of cTnT-positive cells (29). Additionally, cardiac cell sheets formed by reprogrammed cells were implanted in infarcted hearts, leading to cardiac function improvements and decreased adverse cardiac remodeling post MI (44). Although wider investigation is needed, such early studies suggested the positive role of electrical stimulation on DR efficiency.

\section{DISCUSSION}

Nowadays, MI still remains one of the leading causes of death worldwide. Hence, strategies for the replacement of CM loss are of primary interest in regenerative medicine.

DR of human fibroblasts into iCMs might represent a new therapeutic option for myocardial regeneration in addition to cell therapies with iPSC-CMs. Indeed, iPSC-CMs can now be obtained with high efficiency, although their maturation level is generally low, resembling fetal stage CMs (45). Finally, therapies using cells differentiated from pluripotent stem cells, such as iPSCs or embryonic stem cells (ESCs), suffer from the risk of teratoma formation (46).

DR could be exploited as a new source for autologous CMs derived from trans-differentiation of patients' fibroblasts with the advantage of low-to-null tumorigenicity risk if obtained by nonviral methods $(9,20)$. Current research efforts are addressed to increase DR yield and to approach a more adult iCM phenotype. However, one disadvantage of iCMs use in cell therapy is the need for high amounts of patients' fibroblasts (in which potential for in vitro expansion is reduced with respect to stem cells) to generate the required quantities of CMs (from tens to hundreds of millions).

With respect to iPSC-derived technologies, miRcombomediated DR also paves the way to new cell-free in situ strategies for cardiac regeneration, based on the local injection of reprogramming agents able to induce DR of CFs of fibrotic areas or their boundaries into iCMs. Non-viral approaches for in situ DR are safer than viral vectors, in which use is limited by possible off-target effects, mutagenesis risk for integrative virus (retrovirus and lentivirus), and strong immune response (47). Among non-viral strategies for cardiac DR, in vivo administration of small molecule combinations is complicated by the need to locally treat CFs with many drugs (up to nine small molecules) at specific relative concentrations $(48,49)$. On the contrary, the approach based on transient transfection of fibroblasts with miRcombo requires efficient simultaneous release of four miRNAs (miR-1, miR-133, miR208, and miR-499) to CF cytoplasm. For efficient in vivo DR, nanocarrier surface could be functionalized with selected ligands for $\mathrm{CF}$ recognition, coupled to anti-fouling molecules (e.g., ethylene glycol oligomers) to ensure drug delivery specificity in vivo. Additionally, studies on optimal miRcombo dose and delivery kinetics into fibroblast cytoplasm are missing, while it would be fundamental to design efficient miRcombo delivery systems.

Although arrhythmic events have not been reported by reports on in situ cardiac DR in mouse models, this risk could potentially arise from the initial immaturity of early iCMs and be minimized by reducing DR time needed for effective CFs DR into mature iCMs (50). Additionally, based on very recent findings on iPSCCMs therapies, administration of antiarrhythmic drugs could be considered and studied to assist early DR phases, enhancing patients' safety (51).

Another important feature of cardiac DR is its decreasing efficiency as a function of fibroblast aging (52). Scientific literature reported that embryonic vs. adult fibroblasts have 
higher chances for conversion due to an open chromatin conformation (52). However, most DR studies employed embryonic, fetal, or post-natal mouse fibroblasts with superior transdifferentiation ability (52). Importantly, in vitro studies with mouse cells also provide specie-specific outcomes with limited relevance and predictivity for humans. As an exception, pig CFs might be employed considering their close features to human CFs (53). However, investigation on AHCFs is preferred in the perspective of future clinical translation of the approach. In this regard, DR is affected by "patient specificity", as its efficiency may vary significantly, depending on patients' age, sex, and genetic background. Overall safe and efficient standardized protocols taking into account patients' specificity should be defined, allowing more efficient cardiac DR based on clinical cases (52).

Currently, DR research is still at its basic steps. Hence, thorough in vitro studies are demanded, elucidating the role of biochemical and biophysical factors on DR efficiency of AHCFs into iCMs. Based on early findings (26), understanding and controlling the biochemical and biophysical cues of 3D culture substrates are the key for the design of instructive microenvironments improving DR efficiency and fostering the generation of mature iCMs (Table 2). Optimal 3D substrates should mimic cardiac tissue-like stiffness, composition, and architecture (Table 1). However, cell remodeling progressively alters the composition, permeability, and stiffness of $3 \mathrm{D}$ culture matrices, providing dynamic spatiotemporal cues affecting cell fate (54). New advanced techniques able to monitor $3 \mathrm{D}$ cell cultures could unravel the effects of dynamic microenvironmental changes on DR outcomes $(55,56)$. Such interdisciplinary research could be beneficial for efficient DR of human adult fibroblasts into iCMs, given their high epigenetic resistance to phenotype switch. Properly selected types and doses of anti-inflammatory and anti-fibrotic soluble factors could also

\section{REFERENCES}

1. Roger VL. Epidemiology of heart failure. Circ Res. (2013) 113:646-59. doi: 10.1161/CIRCRESAHA.113.300268

2. Segers VFM, Lee RT. Stem-cell therapy for cardiac disease. Nature. (2008) 451:937-42. doi: 10.1038/nature06800

3. Madonna R, Van Laake LW, Davidson SM, Engel FB, Hausenloy DJ, Lecour S, et al. Position paper of the european society of cardiology working group cellular biology of the heart: cell-based therapies for myocardial repair and regeneration in ischemic heart disease and heart failure. Eur Heart J. (2016) 37:1789-98. doi: 10.1093/eurheartj/ ehw113

4. Paoletti C, Divieto C, Chiono V. Impact of biomaterials on differentiation and reprogramming approaches for the generation of functional cardiomyocytes. Cells. (2018) 7:114. doi: 10.3390/cells7090114

5. Engel JL, Ardehali R. Direct cardiac reprogramming: progress and promise. Stem Cells Int. (2018) 2018:1435746. doi: 10.1155/2018/1435746

6. Jayawardena TM, Egemnazarov B, Finch EA, Zhang L, Payne JA, Pandya $\mathrm{K}$, et al. MicroRNA-mediated in vitro and in vivo direct reprogramming of cardiac fibroblasts to cardiomyocytes. Circ Res. (2012) 110:1465-73. doi: 10.1161/CIRCRESAHA.112.269035

7. Paoletti C, Divieto C, Tarricone G, Di Meglio F, Nurzynska D, Chiono V. MicroRNA-mediated direct reprogramming of human adult fibroblasts help in suppressing fibroblast signature, to address the intrinsic epigenetic resistance of adult fibroblasts. Furthermore, more in-depth investigations of the effects of mechanical and electrical stimulations on DR yield and ICM maturation are still rather limited and deserve future attention.

\section{CONCLUSIONS}

The discovery of key biochemical and biophysical factors enhancing cardiac DR and the design of effective and safe nanocarriers for targeted miRcombo delivery will result in significant progresses of both in vitro and in situ cardiac DR approaches, fostering technological advances toward the future clinical application of cardiac DR strategies. However, full exploitation of DR potentialities requires an intense interdisciplinary research, in which bioengineering studies play a key role for the full exploitation of the potentialities of this new emerging approach.

\section{AUTHOR CONTRIBUTIONS}

The manuscript was conceived and written by $\mathrm{CP}$ and VC. VC supervised the project and acquired the funding. Both authors have given approval to the final version of the manuscript.

\section{FUNDING}

This work has received funding from the European Research Council (ERC) under the European Union's Horizon 2020 Research and Innovation Programme (Grant Agreement No. 772168), through BIORECAR ERC Consolidator project (www.biorecar.polito.it).

toward cardiac phenotype. Front Bioeng Biotechnol. (2020) 8:529. doi: 10.3389/fbioe.2020.00529

8. Ieda M, Fu JD, Delgado-Olguin P, Vedantham V, Hayashi Y, Bruneau BG, et al. Direct reprogramming of fibroblasts into functional cardiomyocytes by defined factors. Cell. (2010) 142:375-86. doi: 10.1016/j.cell.2010. 07.002

9. Fu Y, Huang C, Xu X, Gu H, Ye Y, Jiang C, et al. Direct reprogramming of mouse fibroblasts into cardiomyocytes with chemical cocktails. Cell Res. (2015) 25:1013. doi: 10.1038/cr.2015.99

10. Wery M, Kwapisz M, Morillon A. Noncoding RNAs in gene regulation. Wiley Interdiscip Rev Syst Biol Med. (2011) 3:728-38. doi: 10.1002/ wsbm.148

11. Batty JA, Lima JAC, Kunadian V. Direct cellular reprogramming for cardiac repair and regeneration. Eur J Heart Fail. (2016) 18:145-56. doi: 10.1002/ejhf.446

12. Nam Y-J, Song K, Olson EN. Heart repair by cardiac reprogramming. Nat Med. (2013) 19:413-5. doi: 10.1038/nm.3147

13. Hodgkinson CP, Kang MH, Dal-Pra S, Mirotsou M, Dzau VJ. MicroRNAs and cardiac regeneration. Circ Res. (2015) 116:1700-11. doi: 10.1161/CIRCRESAHA.116.304377

14. Jayawardena TM, Finch EA, Zhang L, Zhang H, Hodgkinson CP, Pratt RE, et al. MicroRNA induced cardiac reprogramming in vivo. Circ Res. (2015) 116:418-24. doi: 10.1161/CIRCRESAHA.116.304510 
15. Nam Y-J, Song K, Luo X, Daniel E, Lambeth K, West K, et al. Reprogramming of human fibroblasts toward a cardiac fate. Proc Natl Acad Sci USA. (2013) 110:5588-93. doi: 10.1073/pnas.1301019110

16. Zhao Y, Londono P, Cao Y, Sharpe EJ, Proenza C, O'Rourke R, et al. High-efficiency reprogramming of fibroblasts into cardiomyocytes requires suppression of pro-fibrotic signalling. Nat Commun. (2015) 6:8243. doi: $10.1038 /$ ncomms 9243

17. Kang $\mathrm{MH}$, Hu J, Pratt RE, Hodgkinson CP, Asokan A, Dzau VJ. Optimizing delivery for efficient cardiac reprogramming. Biochem Biophys Res Commun. (2020) 533:9-16. doi: 10.1016/j.bbrc.2020. 08.104

18. Lee SWL, Paoletti C, Campisi M, Osaki T, Adriani G, Kamm RD, et al. MicroRNA delivery through nanoparticles. J Control Release. (2019) 313:80-95. doi: 10.1016/j.jconrel.2019.10.007

19. Muniyandi P, Palaninathan V, Mizuki T, Maekawa T, Hanajiri T, Mohamed MS. Poly(lactic- co -glycolic acid)/polyethylenimine nanocarriers for direct genetic reprogramming of MicroRNA targeting cardiac fibroblasts. ACS Appl Nano Mater. (2020) 3:2491-505. doi: 10.1021/acsanm. $9 \mathrm{~b} 02586$

20. Yang L, Xue S, Du M, Lian F. Highly efficient MicroRNA delivery using functionalized carbon dots for enhanced conversion of fibroblasts to cardiomyocytes. Int J Nanomed. (2021) 16:3741-54. doi: 10.2147/IJN.S3 04873

21. Passaro F, Tocchetti CG, Spinetti G, Paudice F, Ambrosone L, Costagliola C, et al. Targeting fibrosis in the failing heart with nanoparticles. Adv Drug Deliv Rev. (2021) 174:461-81. doi: 10.1016/j.addr.2021.05.004

22. Bejleri D, Davis ME. Decellularized extracellular matrix materials for cardiac repair and regeneration. Adv Healthc Mater. (2019) 8:1801217. doi: 10.1002/ adhm.201801217

23. McCain ML, Lee H, Aratyn-Schaus Y, Kléber AG, Parker KK. Cooperative coupling of cell-matrix and cell-cell adhesions in cardiac muscle. Proc Natl Acad Sci USA. (2012) 109:9881-6. doi: 10.1073/pnas.12030 07109

24. Olvera D, Molina MS, Hendy G, Monaghan MG. electroconductive melt electrowritten patches matching the mechanical anisotropy of human myocardium. Adv Funct Mater. (2020) 30:1909880. doi: 10.1002/ adfm. 201909880

25. MacQueen LA, Sheehy SP, Chantre CO, Zimmerman JF, Pasqualini FS, Liu X, et al. A tissue-engineered scale model of the heart ventricle. Nat Biomed Eng. (2018) 2:930-41. doi: 10.1038/s41551-018-0271-5

26. Li Y, Dal-Pra S, Mirotsou M, Jayawardena TM, Hodgkinson CP, Bursac N, et al. Tissue-engineered 3-dimensional (3D) microenvironment enhances the direct reprogramming of fibroblasts into cardiomyocytes by microRNAs. Sci Rep. (2016) 6:38815. doi: 10.1038/srep38815

27. Sia J, Yu P, Srivastava D, Li S. Effect of biophysical cues on reprogramming to cardiomyocytes. Biomaterials. (2016) 103:1-11. doi: 10.1016/ j.biomaterials.2016.06.034

28. Kurotsu S, Sadahiro T, Fujita R, Tani H, Yamakawa H, Tamura F, et al. Soft matrix promotes cardiac reprogramming via inhibition of YAP/TAZ and suppression of fibroblast signatures. Stem Cell Rep. (2020) 15:612-28. doi: 10.1016/j.stemcr.2020.07.022

29. Song SY, Yoo J, Go S, Hong J, Su Sohn H, Lee JR, et al. Cardiac-mimetic cell-culture system for direct cardiac reprogramming. Theranostics. (2019) 9:6734-44. doi: 10.7150/thno.35574

30. Smith AW, Hoyne JD, Nguyen PK, McCreedy DA, Aly H, Efimov IR, et al. Direct reprogramming of mouse fibroblasts to cardiomyocyte-like cells using Yamanaka factors on engineered poly(ethylene glycol) (PEG) hydrogels. Biomaterials. (2013) 34:6559. doi: 10.1016/j.biomaterials.2013. 05.050

31. Zhou H, Morales MG, Hashimoto H, Dickson ME, Song $\mathrm{K}$, Ye W, et al. ZNF281 enhances cardiac reprogramming by modulating cardiac and inflammatory gene expression. Genes Dev. (2017) 31:1770-83. doi: $10.1101 / \operatorname{gad} .305482 .117$

32. Testa G, Russo M, Di Benedetto G, Barbato M, Parisi S, Pirozzi F, et al. Bmil inhibitor PTC-209 promotes Chemicallyinduced Direct Cardiac Reprogramming of cardiac fibroblasts into cardiomyocytes. Sci Rep. (2020) 10:1-16. doi: 10.1038/s41598-02063992-8
33. Muraoka N, Nara K, Tamura F, Kojima H, Yamakawa H, Sadahiro T, et al. Role of cyclooxygenase-2-mediated prostaglandin E2-prostaglandin E receptor 4 signaling in cardiac reprogramming. Nat Commun. (2019) 10:1-5. doi: 10.1038/s41467-019-08626-y

34. Yamakawa H, Muraoka N, Miyamoto K, Sadahiro T, Isomi M, Haginiwa $\mathrm{S}$, et al. Fibroblast growth factors and vascular endothelial growth factor promote cardiac reprogramming under defined conditions. Stem Cell Rep. (2015) 5:1128-42. doi: 10.1016/j.stemcr.2015.10.019

35. Ifkovits JL, Addis RC, Epstein JA, Gearhart JD. Inhibition of TGF $\beta$ signaling increases direct conversion of fibroblasts to induced cardiomyocytes. PLoS ONE. (2014) 9:e89678. doi: 10.1371/journal.pone.0089678

36. Bunney PE, Zink AN, Holm AA, Billington CJ, Kotz CM Orexin activation counteracts decreases in nonexercise activity thermogenesis (NEAT) caused by high-fat diet. Physiol Behav. (2017) 176:139-48. doi: 10.1016/j.physbeh.2017.03.040

37. Jin Y, Lee JS, Kim J, Min S, Wi S, Yu JH, et al. Three-dimensional brain-like microenvironments facilitate the direct reprogramming of fibroblasts into therapeutic neurons. Nat Biomed Eng. (2018) 2:522-39. doi: 10.1038/s41551-018-0260-8

38. Sauls K, Greco TM, Wang L, Zou M, Villasmil M, Qian L, et al. Initiating events in direct cardiomyocyte reprogramming. Cell Rep. (2018) 22:1913-22. doi: 10.1016/j.celrep.2018.01.047

39. Nian $M$, Lee $P$, Khaper $N$, Liu P. Inflammatory cytokines and postmyocardial infarction remodeling. Circ Res. (2004) 94:1543-53. doi: 10.1161/01.RES.0000130526.20854.fa

40. LaBarge W, Mattappally S, Kannappan R, Fast VG, Pretorius D, Berry JL, et al. Maturation of three-dimensional, hiPSC-derived cardiomyocyte spheroids utilizing cyclic, uniaxial stretch and electrical stimulation. PLoS ONE. (2019) 14:e0219442. doi: 10.1371/journal.pone.0219442

41. Occhetta P, Isu G, Lemme M, Conficconi C, Oertle P, Räz C, et al. A threedimensional in vitro dynamic micro-tissue model of cardiac scar formation. Integr Biol. (2018) 10:174-83. doi: 10.1039/C7IB00199A

42. Machiraju P, Greenway SC. Current methods for the maturation of induced pluripotent stem cell-derived cardiomyocytes. World J Stem Cells. (2019) 11:33. doi: 10.4252/wjsc.v11.i1.33

43. Ronaldson-Bouchard K, Ma SP, Yeager K, Chen T, Song L, Sirabella $\mathrm{D}$, et al. Advanced maturation of human cardiac tissue grown from pluripotent stem cells. Nature. (2018) 556:239-43. doi: 10.1038/s41586-018-0 016-3

44. Song SY, Kim H, Yoo J, Kwon SP, Park BW, Kim J, et al. Prevascularized, multiple-layered cell sheets of direct cardiac reprogrammed cells for cardiac repair. Biomater Sci. (2020) 8:4508-20. doi: 10.1039/D0BM0 0701C

45. Ulmer BM, Eschenhagen T. Human pluripotent stem cell-derived cardiomyocytes for studying energy metabolism. Biochim Biophys Acta Mol Cell Res. (2020) 1867:118471. doi: 10.1016/j.bbamcr.2019. 04.001

46. Yamanaka S. Pluripotent stem cell-based cell therapy-promise and challenges. Cell Stem Cell. (2020) 27:523-31 doi: 10.1016/j.stem.2020. 09.014

47. Mitchell MJ, Billingsley MM, Haley RM, Wechsler ME, Peppas NA, Langer R. Engineering precision nanoparticles for drug delivery. Nat Rev Drug Discov. (2020) 20:101-24. doi: 10.1038/s41573-020-0090-8

48. Huang C, Tu W, Fu Y, Wang J, Xie X. Chemical-induced cardiac reprogramming in vivo. Cell Res. (2018) 28:686-9. doi: 10.1038/ s41422-018-0036-4

49. Cao N, Huang Y, Zheng J, Spencer CI, Zhang Y, Fu JD, et al. Conversion of human fibroblasts into functional cardiomyocytes by small molecules. Science. (2016) 352:1216-20. doi: 10.1126/science.aaf1502

50. Sadahiro T, Yamanaka S, Ieda M. Direct cardiac reprogramming. Circ Res. (2015) 116:1378-91. doi: 10.1161/CIRCRESAHA.116.305374

51. Nakamura K, Neidig LE, Yang X, Weber GJ, El-Nachef D, Tsuchida $\mathrm{H}$, et al. Pharmacologic therapy for engraftment arrhythmia induced by transplantation of human cardiomyocytes. Stem Cell Rep. (2021) 16:1-15. doi: 10.1016/j.stemcr.2021.08.005

52. Wang H, Yang Y, Liu J, Qian L. Direct cell reprogramming: approaches, mechanisms and progress. Nat Rev Mol Cell Biol. (2021) 22:410-24. doi: 10.1038/s41580-021-00335-Z 
53. Singh VP, Mathison M, Patel V, Sanagasetti D, Gibson BW, Yang J, et al. MiR-590 promotes transdifferentiation of porcine and human fibroblasts toward a cardiomyocyte-like fate by directly repressing specificity protein 1. J Am Hear Assoc Cardiovasc Cerebrovasc Dis. (2016) 5:e003922. doi: 10.1161/JAHA.116.003922

54. Madl CM, LeSavage BL, Dewi RE, Dinh CB, Stowers RS, Khariton M, et al. Maintenance of neural progenitor cell stemness in $3 \mathrm{D}$ hydrogels requires matrix remodelling. Nat Mater. (2017) 16:1233-42. doi: 10.1038/nma t5020

55. Mortati L, Divieto C, Sassi MP. CARS and SHG microscopy to follow collagen production in living human corneal fibroblasts and mesenchymal stem cells in fibrin hydrogel 3D cultures. J Raman Spectrosc. (2012) 43:675-80. doi: $10.1002 /$ jrs.3171

56. Loebel C, Mauck RL, Burdick JA. Local nascent protein deposition and remodelling guide mesenchymal stromal cell mechanosensing and fate in three-dimensional hydrogels. Nat Mater. (2019) 18:883-91. doi: 10.1038/s41563-019-0307-6
Conflict of Interest: The authors declare that the research was conducted in the absence of any commercial or financial relationships that could be construed as a potential conflict of interest.

Publisher's Note: All claims expressed in this article are solely those of the authors and do not necessarily represent those of their affiliated organizations, or those of the publisher, the editors and the reviewers. Any product that may be evaluated in this article, or claim that may be made by its manufacturer, is not guaranteed or endorsed by the publisher.

Copyright (C) 2021 Paoletti and Chiono. This is an open-access article distributed under the terms of the Creative Commons Attribution License (CC BY). The use, distribution or reproduction in other forums is permitted, provided the original author(s) and the copyright owner(s) are credited and that the original publication in this journal is cited, in accordance with accepted academic practice. No use, distribution or reproduction is permitted which does not comply with these terms. 